\title{
Sentence structure study of Emoji communication
}

\author{
Qiu Menglan \\ International Business School \\ Yunnan University of Finance and Economic \\ Kunming, China \\ 879579662@qq.com \\ Feng Yuhui * \\ International Business School
}

\author{
Yunnan University of Finance and Economic \\ Kunming, China \\ feng@ynufe.edu.cn \\ Yang Haiyang \\ International Business School \\ Yunnan University of Finance and Economic \\ Kunming, China \\ frank2h@foxmail.com
}

\begin{abstract}
June 22, 2015, Chevrolet sent out a press release which is completely made up of Emoji symbols. Once this press release was published, it immediately drew a massive attention of Internet users who intend to interpret it. Unfortunately, there are only a few interpretations were corresponded with the results published by the company. This study analyzed the failure of the interpretation, and improved it from an Emoji-statement perspective in accordance with rules from cognitive psychology and linguistics. Network experiment was conducted later to see if this method is effective. The results showed that by adopting specific Emoji syntactic structures and rules, subjects' expression on Emoji statement can be more accurate and the problems in signifier and referent of readers can be resolve.
\end{abstract}

Keywords—emoji; sentence structure; semiology

\section{INTRODUCTION}

The advent of the Internet era has undoubtedly popularized online social chat, during this process, some simple and interesting Emoji symbols have been integrated into the Internet world. In an online communication, Emoji symbols work better for demonstrating emotional changes in different scenarios, besides, many symbols can convey various meanings other than what they are normally appealed to be. Masahide, Yuasa argued that nonverbal graphic symbols are essentially network-assisted means of communication [1]. In addition, nonverbal graphic symbols are a form of language that rarely suffers from communication disorders [2]. In the process of cross-cultural communication, pictures or symbols can break through the language barrier in information transmission in most cases [3], thus make up for national or regional differences. Experiments confirmed that there is no cross-cultural or cross-regional identification obstacles in the use of Emoji, and can play a role in auxiliary communication in some special scenes [4]. Therefore, many young people began to use Emoji frequently instead of the text expression. Compared to the traditional way of communication, Using Emoji not only makes the Internet more graphical, but also offers a more vivid and accurate way of expressing people's thoughts and emotions.

On June 22, 2015, Chevrolet released a new car targeted at young users (http://madbrief.com/archives/18262). In order to gain attentions and communicate with their customers, the promotion team choose a new way to introduce their car, which consist of a press release completely made of Emoji (fig. 1.). Once this press release was published, it quickly attracted massive attention, numerous Emoji heavy users have participated in the decoding activities and enjoying themselves.

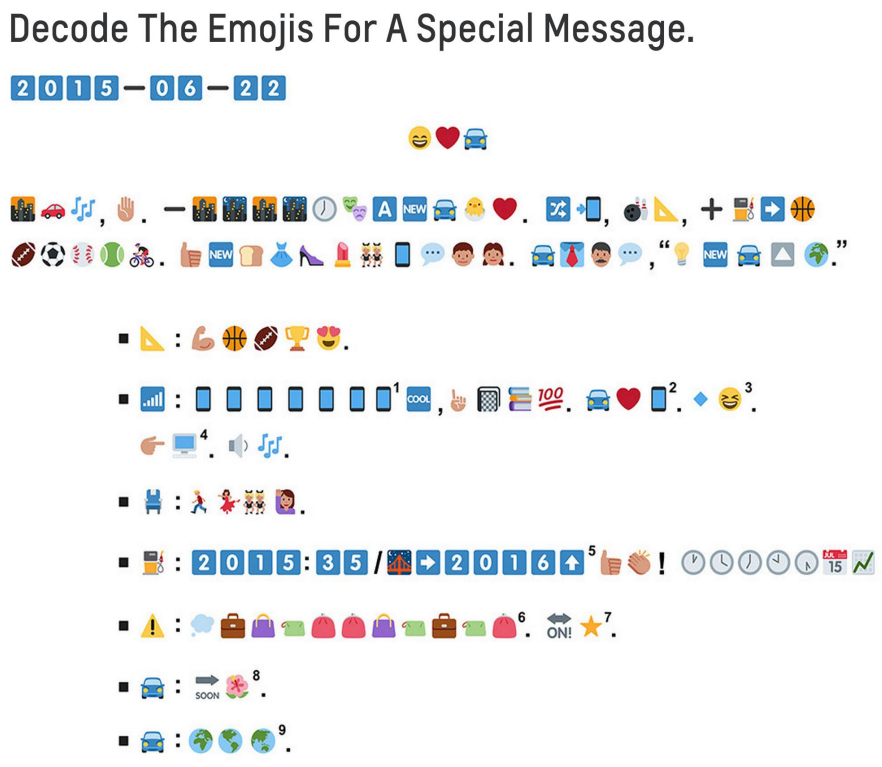

国

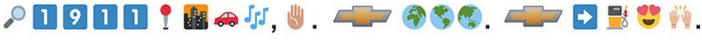 a $\square$ www.chevrolet.com.}

Fig. 1. The press release completely made of Emoji

But the results of user's interpretation were found unsatisfactory. The official version of interpretation (shown in fig. 2.) showed there is only a small portion of people who have done a perfect work. The results surely lead us to think deeper on how to improve the recognition rate between the publisher and the reader.

* Corresponding author 
DETROIT - In two days at the Fillmore Theatre at 7 p.m., a new Cruze will be born and you are going to love it:

The all-new 2016 Cruze blends innovative technology, striking design and impressive efficiency into one sporty ride. It's the best new thing since sliced bread for stylish and socially connected people. A Chevrolet spokesperson said: "We had the idea that the new Cruze could change the world,"

\section{- Design: Athletic build, stylish and good looking}

- Technology: 7 connected devices with avallable 4G LTE WI-FI. Cooll First in its class to offer compatibility with Apple CarPlay and Android Auto. Bluetooth compatible. Touch screen. Speakers/music.

- Seating: Seats 5

- Fuel efficiency: The 2015 Cruze offers an EPA-estimated $35 \mathrm{mpg}$ hwy. The 2016 Cruze is expected to offer better fuel economy, which is awesome. Look at how much time will pass before you need to fill up your tank again!

- Safety: 10 air bags. Comes with OnStar standard for 6 months.

- Avallable; Soonl Coming in Spring 2016

- Available: Globally

In conclusion, get ready to go places in your Cruze. It has the technology and fuel efficiency you need wrapped up in a fun ride. Prepare to fall in love.

Follow \#ChevyGoesEmoji to learn more and join the fun!

Founded in 1911 in Detroit, Chevrolet is now one of the world's largest car brands, doing business in more than 115 countries and selling around 4.8 million cars and trucks a year. Chevrolet provides customers with fuel-efficient vehicles that feature engaging performance, design that makes the heart beat, passive and active safety features and easy-to-use technology, all at a value. More information on Chevrolet models can be found at http://www. chevrolet.com.

Fig. 2. The official version of interpretation

Emoji is a visual emotion symbol used in wireless communication devices created by a Japanese called Shigetaka Kurita. A symbol is a sign or mark that represents a certain thing or expresses a meaning, and is composed of signifier and referent. Signifier is a specific object (the symbol itself), referent refers to the psychological concept (the content of symbol) [5]. A symbol is the combination of signifier and referent, its role lies in the transfer of message [6]. In the transmission, the coding process refers to the composition process of a letter. Specifically, in the visual communication of a symbol, the sender's end does the encoding process, which is to transform abstract information into a perceptible message via specific rules. The recipient side of the process is called decoding. The user reconstruct the information according to their own understanding of the message [7]. It requires the perfect combination of the signifier and the referent to fully express what the symbol means.

Using Emoji instead of writing exclusively to communicate will create various problems such as low interpretation accuracy. This is probably because most Emoji users simply use Emoji as a substitute for particular emotion and combine it with the text to convey their intention. The exclusive use of Emoji in a sentence have not been widely adopted so far. The Emoji grammatical structure is not used properly in the process of interpreting and lead to inaccurate interpretation toward the statements. Therefore, the rational use of Emoji grammatical structure can greatly eliminate the problems in signifier and referent, and improve user's correct rate in their interpretation of Emoji sentence.

This study aims to run a data analysis and an improved experiment on Chevrolet's ad. By doing a data analysis, users' interpretations will be collected and processed, each interpreted statement will be given a score based on the accuracy of interpretation, and the average score for each statement will be calculated for analyzing the grammatical structure in each statement. The improved experiment will firstly distinguish statements with relatively lower recognition and modify their grammatical structure in accordance with cognitive psychology and linguistics rules. Then a further experiment will be conducted to determine whether the improved method is effective. This will eventually lead to the conclusion that certain rules are capable of improving the recognition results on Emoji statements.

\section{METHODS}

Chevrolet's Emoji ad introduce its new Cruze in 8 aspects including appearance, technology, fuel consumption, security, etc. We collected the interpretations from the Internet and studied how they were interpreted.

First, we split the press release into statements and have them numbered.

We then use the typical five-level Likert-type scale (from strongly disagree to strongly agree) to measure how the statements were interpreted.

From strongly disagree to strongly agree, we rated them in a score range from 1 to 5 and caculated the average score for each statement.

\section{A. Data collection}

We search for interpretations of the Chevrolet Emoji ad on the Internet and analyzed all the information gathered.

\section{B. Data analysis}

The average score for each statement is shown in Table I below.

TABLE I. THE AVERAGE SCORE FOR EACH STATEMENT

\begin{tabular}{|c|c|c|}
\hline Num & Emoji Statement. & Average Score \\
\hline A01 & A $९$ त्रि & 4.55 \\
\hline A02 & 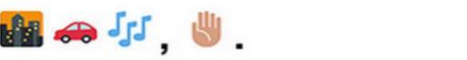 & 4.1 \\
\hline A03 & 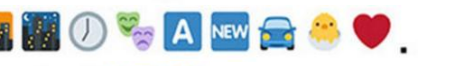 & 4.1 \\
\hline A04 & 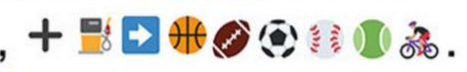 & 3.5 \\
\hline A05 & 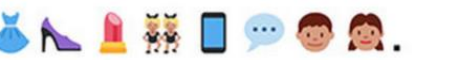 & 3.7 \\
\hline
\end{tabular}




\begin{tabular}{|c|c|}
\hline A06 & 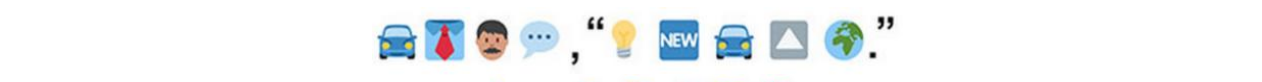 \\
\hline A07 & $. \Delta: 6400$ \\
\hline A08 & 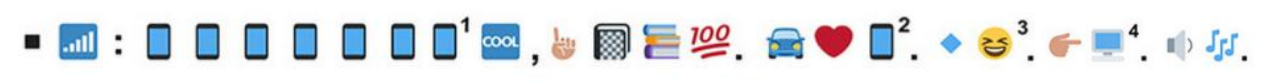 \\
\hline A09 & 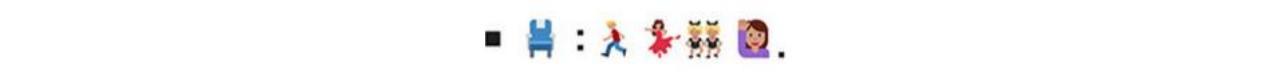 \\
\hline A10 & 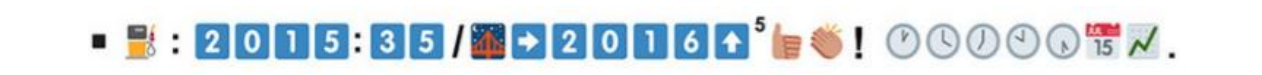 \\
\hline A11 & - ! : 불ㅇำ \\
\hline A12 & - $: \underset{\text { soon }}{\Rightarrow} \mathbb{s}^{8}$ \\
\hline A13 & - \\
\hline A14 & 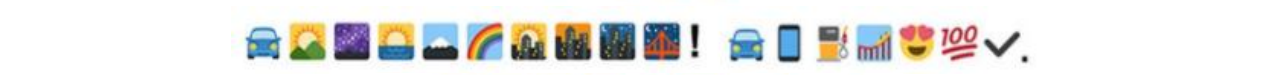 \\
\hline $\mathbf{A 1 5}$ & 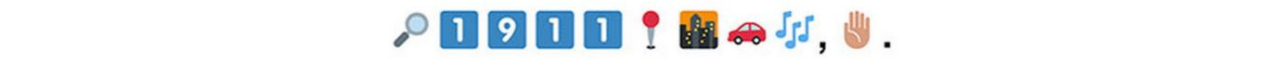 \\
\hline A16 & ¿468. \\
\hline A17 & 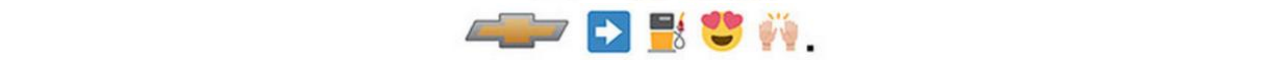 \\
\hline $\mathbf{A 1 8}$ & (2) $\square$ www.chevrolet.com. \\
\hline
\end{tabular}

\section{Discussion}

The above results showed there is a big difference among the average score for each statement. Statement A18 reached the highest score, which is 5 , meaning that it can be interpreted correctly by all the users. Statement A06 reached the lowest score of 2.5, which means only half of the users did the right interpretation. It can be concluded that statements with clear grammatical structure achieved higher score than those without.

\section{EXPERIMENT}

According to previous findings, we modified statements with relative lower score (A06, A07, A08, A09) on their grammatical structure and formed into new Emoji statements. Then a modified experiment was launched on the Internet and new interpretations were collected.

\section{A. Subjects}

Users from Weibo, WeChat, Q-zone, Twitter, etc.

\section{B. experimental design}

1) Use verbic graphic symbols when expressing a person's behavior or thought. For example, in statement A06, we can use indicate "Talk".

2) Try to use symbols which are cognitive and normative. In statement A08, * can be used for "Bluetooth" and $\cong$ is for "Wi-Fi".

3) Use direct numbers instead of simply accumulation of symbols when trying to show the quantity of something. For example, in statement A09, it's better to use seats" and $\square * 7$ for "Seven cellphones".

4) Use relational graphic symbols to indicate the relationship between several things. In statement A08, $\left\{\subset \&{ }^{1}\right.$ \} can be used to indicate "Compatible with Apple CarPlay system and Android system, and in statement A07, we

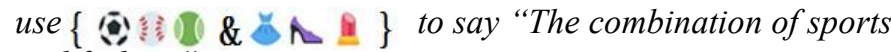
and fashion".

\section{Statistical analysis}

Based on the grammatical structures mentioned above, we modified statements A06, A07, A08, A09. Result of the modified experiment is shown in Table II .

TABLE II. THE AVERAGE SCORE FOR MODIFIED STATEMENTS

\begin{tabular}{|c|c|c|}
\hline Number & Modified Statements & Average Score \\
\hline N06 & बिए & 4.3 \\
\hline N07 & $\Delta:\{6 \div 0 \& 8 n$ & 4.2 \\
\hline N08 & 尺 : $\square * \sqrt{7},\left\{@ \& \mathbb{N}^{\prime}\right\}$. & (1) S Sj". \\
\hline N09 & 菂: $\frac{1}{2} * 5$ & 4.4 \\
\hline
\end{tabular}

D. discussion

The result has shown that the modified Emoji statements N09 reached the highest score of 4.4, Statement N07 reached the lowest score of 4.2. the modified Emoji statements N06, N07, N08, N09 reached a higher average score on their interpretation than their counterparts.

\section{Using the TEMPLATE ANALYSES AND RESUlts}

Compare the results with the previous data, as shown in table III.

TABLE III. AVERAGE SCORE OF 06、07、08、09

\begin{tabular}{lllll}
\hline Number & $\mathbf{0 6}$ & $\mathbf{0 7}$ & $\mathbf{0 8}$ & $\mathbf{0 9}$ \\
\hline Before & 2.5 & 2.55 & 2.7 & 2.6 \\
After & 4.3 & 4.2 & 4.25 & 4.4 \\
\hline
\end{tabular}


Use EXCEL software to process the data of two sets, and the test of data was carried out by T-test of the two-sample mean of the heteroscopanova. The significant degree of alpha $=0.05$. The analysis results are shown in table IV .

TABLE IV. THE ANALYSIS RESULTS BY T-TEST

\begin{tabular}{lll}
\hline Analysis & Before & After \\
\hline Average & 2.5875 & 4.2875 \\
Variance & 0.007292 & 0.007292 \\
Observation value & 4 & 4 \\
Assumed mean difference & 0 \\
df & 6 \\
t Stat & & -28.1547 \\
$\mathbf{P}(\mathbf{T}<=$ t) & $1.33 \mathrm{E}-07$ \\
$\mathbf{T}$ double tail critical & 2.446912 \\
\hline
\end{tabular}

The results showed that $\mathrm{P}=1.33 \mathrm{E}-07$. By looking up the table, we think they are significant differences if $\mathrm{P}<0.05$.

Therefore, there are significant differences between the two sets of data, and the experimental improvement method is effective.

\section{DISCUSSION AND CONCLUSION}

This study showed that the improved Emoji statements are able to be correctly interpreted by users. The average score on interpretation was significantly higher after our modification. By using Emoji grammatical rules properly, users are able to convey a more accurate expression with Emoji statements and narrow both the signifier and referent gap in transmission.
Owning to the limitation of space, the above experiment only made a few simple emoji rules. Readers are welcome to conduct a more complete research combined the ideas provided in this article.

\section{ACKNOWLEDGMENTS}

This research was sponsored by the National Natural Science Foundation of China (61662084) and Science Research Foundation of Yunnan Provincial Department of Education（2017YJS008） commissioned by China's Ministry of Education.

\section{REFERENCES}

[1] Masahide, Yuasa, Keiichi, Saito, Naoki \& Mukawa, "Emoticons Convey Emotions without Cognition of Faces: an FMRI study," Conference on Human Factors in Computing Systems Canada 2006, New York, ACM, 2006, pp. 1564-1570.

[2] M.J. Zhang, "Symbolic regression of interpersonal communication -factor analysis of network emoticons Emoji in social media explosion". Journalism Lover, No.45612, 2015, pp. 47-51.

[3] G.W. Yu, and Y. Qin, "A tentative study of network emoticons in Linguistic Perspective," Journal of Graduate School of Chinese Academy of Social Sciences, No.18101, 2011, pp. 130-135.

[4] Y.H. Feng, M.L. Qiu, Y. Li, and H.Y. Yang, "Cross-culture Business Communication by Emoji in GMS," The First International Symposium on Business Cooperation and Development in South-East and South Asia under B\&R Initiative (ISBCD-16), 2016, pp. 181-186.

[5] S. Hoare, "What does this mean?-the 75 basic concepts of Semiotics," Beijing: Central Compilation and publication press, 2010.

[6] R.X. Zhang, and X.Y. Geng, "A semiotic examination of industrial design," Packing Engineering, vol. 23, 2002, pp. 123-125.

[7] R.X. Zhang, "Industrial design concepts and methods," Beijing: Beijing Institute of Technology press, 1996. 